\section{Asymmetric Arylation of Secondary Alkyl Electrophiles}

Metal-Catalyzed Asymmetric

Synthesis and

Stereoselective

Reactions

\section{Key words}

bis(oxazoline)s

Negishi arylation

nickel

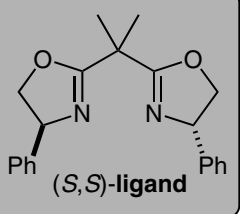

$\mathrm{NiCl}_{2} \cdot$ glyme (6 mol\%)<smiles>CC(=O)C(Br)Br</smiles>

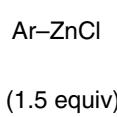

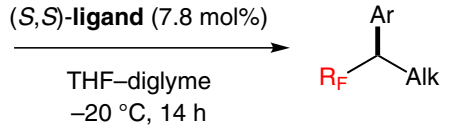

Selected examples:<smiles>COc1ccccc1C(CCc1ccccc1)C(F)(F)F</smiles>

$50 \%$ yield, $87 \%$ ee ( $40 \mathrm{~h}$ reaction time)<smiles>COc1cccc(C(C)C(F)(F)F)c1</smiles>

$81 \%$ yield, $91 \%$ ee<smiles>COc1cccc(C(CCc2ccccc2)C(F)(F)F)c1</smiles>

$91 \%$ yield, $96 \%$ ee (gram scale)<smiles>COc1cccc(C(C(F)(F)F)C(Cl)(Cl)Cl)c1</smiles>

$89 \%$ yield, $96 \%$ ee<smiles>FC(F)(F)C(CCc1ccccc1)c1ccccc1</smiles>

$86 \%$ yield, $95 \%$ ee<smiles>COc1cccc(C(C(Br)Br)C(F)(F)F)c1</smiles>

$80 \%$ yield, $96 \%$ ee<smiles>FC(F)(F)C(CCc1ccccc1)c1ccc2occc2c1</smiles>

$60 \%$ yield, $94 \%$ ee<smiles>COc1cccc(C(C(C)(F)F)C(F)(F)F)c1</smiles>

$91 \%$ yield, $97 \%$ ee<smiles></smiles>

$68 \%$ yield, $94 \%$ ee<smiles>COc1cccc(C([AlH2])C(=O)C2CCCCC2)c1</smiles>

$92 \%$ yield, $99 \%$ ee<smiles>COc1cccc([Ge]Cl)c1</smiles>

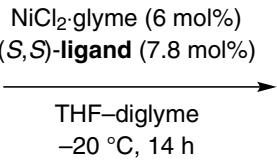<smiles>COc1cccc(C(CCc2ccccc2)C(F)(F)F)c1</smiles>

Significance: Enantiodivergent cross-coupling of an arylzinc reagent and a secondary alkyl halide with a trifluormethyl substituent was achieved by using a readily available nickel/bis(oxazoline) catalyst. The fluorinated products were obtained in good yields and with high enantioselectivities.
sYNFACTS Contributors: Hisashi Yamamoto, Ramesh C. Samanta Synfacts 2015, 11(10), 1077 Published online: 18.09.2015 Dol: 10.1055/s-0035-1560233; Reg-No.: H11715SF
Comment: Fu and co-workers have previously reported an enantiodivergent cross-coupling of a racemic secondary electrophile by using a chiral nickel catalyst (J. Am. Chem. Soc. 2005, 127, 4594; J. Am. Chem. Soc. 2014, 136, 12161). The chiral catalyst can differentiate between a trifluoromethyl and an alkyl group to deliver the crosscoupling product with high enantioselectivity. The cross-coupling reaction is not air-sensitive, as identical results were obtained when the reaction was conducted in the presence of air. 